\title{
A New Paradigm for South Pacific Climate Variability and Predictability
}

\author{
Jiale Lou ( $\sim$ jiale.lou@noaa.gov ) \\ NOAA https://orcid.org/0000-0002-4014-5242 \\ Terence O'Kane \\ CSIRO \\ Neil Holbrook \\ University of Tasmania https://orcid.org/0000-0002-3523-6254
}

\section{Article}

Keywords: climate variability, atmspheric science, ocean science, South Pacific

Posted Date: July 19th, 2021

DOl: https://doi.org/10.21203/rs.3.rs-602270/v1

License: (1) This work is licensed under a Creative Commons Attribution 4.0 International License.

Read Full License

Version of Record: A version of this preprint was published at Communications Earth \& Environment on October 21st, 2021. See the published version at https://doi.org/10.1038/s43247-021-00295-4. 
$4 \quad{ }^{1}$ Institute for Marine and Antarctic Studies, University of Tasmania, Hobart, TAS, Australia

$5{ }^{2}$ ARC Centre of Excellence for Climate System Science, University of Tasmania, Hobart, TAS, Australia 
21 Pacific climate variability is largely understood based on El Niño-Southern Oscillation

22 (ENSO), the North Pacific focused Pacific decadal oscillation (PDO) and/or the whole of

23 Pacific region interdecadal Pacific oscillation - which respectively represent the dominant

24 modes of interannual and decadal climate variability. However, the role of the South Pacific,

25 including atmospheric drivers and cross-scale interactions between interannual and decadal

26 climate variability, has received considerably less attention. Here we propose a new

27 paradigm for South Pacific climate variability whereby the Pacific-South American (PSA)

28 mode, characterised by two mid-tropospheric modes (PSA1 and PSA2), provides coherent noise forcing that acts to excite multiple spatiotemporal scales of oceanic responses in the upper South Pacific Ocean ranging from seasonal to decadal. While PSA1 has long been recognised as highly correlated with ENSO, we find that PSA2 is critically important in generating a sea surface temperature (SST) quadrupole pattern in the extratropical South Pacific. This sets up a precursor that optimally determines the predictability and evolution of SST 9 months in advance of the peak phases of both the leading South Pacific SST mode and ENSO. Our results show that the atmospheric PSA mode is the key driver of oceanic variability in the South Pacific subtropics. Importantly, we anticipate that South Pacific Ocean climate variability is potentially predictable on seasonal to interannual time scales. We expect this new knowledge to be of potential benefit to marine conservation, fisheries and aquaculture management practices that utilise climate forecasts for their operations and 40 planning. 
41 Pacific decadal variability has been historically understood in terms of the North Pacific-focused

42 Pacific decadal oscillation (PDO ${ }^{[1]}$ ) and by the basin-scale interdecadal Pacific oscillation (IPO

$\left.43^{[2]}\right)$. However, neither the PDO nor the IPO explicitly isolate the South Pacific contribution.

44 Analogous to the PDO, the South Pacific decadal oscillation (SPDO ${ }^{[3,4]}$ ) has been identified and

45 described as the South Pacific centre of action contributing to the entire basin-wide Pacific decadal

46 variability. Refs ${ }^{[4,5]}$ show that the SPDO can be viewed as a combination of different dynamical

47 processes operating on different timescales. Those processes include the atmospheric forcing,

48 tropical El Niño-Southern Oscillation (ENSO) teleconnections, and the internal oceanic dynamics.

49 Importantly, the atmospheric forcing associated with the Pacific-South American pattern 1 (PSA1)

50 has been identified as the main stochastic driver of the SPDO ${ }^{[4]}$ (cf Fig.1 (left)). ENSO has been

51 shown to impact extratropical variability via the atmospheric bridge ${ }^{[6]}$ and oceanic pathways ${ }^{[7]}$.

52 Recent studies point out that extratropical ocean dynamics can feed back to the tropical Pacific via

53 a sea surface temperature (SST) quadrupole pattern ${ }^{[8]}$ or a South Pacific meridional mode ${ }^{[9-11]}$.

54 These oceanic processes act as extratropical precursors that might potentially guide the

55 predictability and evolution of ENSO.

56 In this study, we propose a new paradigm for South Pacific climate variability whereby the pair of

57 atmospheric eastward-propagating PSA modes can excite extratropical South Pacific Ocean

58 responses on multiple time scales ranging from seasonal to decadal. Although the South Pacific

59 Ocean responds to fast-varying atmospheric forcing on distinct timescales, the resultant spatial

60 SST features remain difficult to distinguish from the background state. This suggests that the

61 characterisation and identification of predictable signals, and their sources, require careful

62 separation of the climate signal and relevant noise processes. This remains a necessary but

63 challenging problem. 


\section{The atmospheric Pacific-South American mode}

65 The PSA mode is represented by an eastward-propagating wave train extending from eastern Australia to Argentina, characterised in mid-tropospheric geopotential height in terms of two

67 invariant empirical orthogonal function (EOF) patterns with associated principal component (PC) time series (PSA1 and PSA2; see methods section) whose phases are nearly in quadrature with each other and whose explained variances are of nearly equal amplitude ${ }^{[12,13]}$. In combination, they produce the single propagating PSA mode. The PSA mode is known to strongly influence the

71 Antarctic cryosphere ${ }^{[14]}$, wind and significant wave heights across the Southern Hemisphere 72 oceans ${ }^{[13]}$, and the South American monsoon system including rainfall ${ }^{[15]}$ and weather and climate 73 extremes over Brazil ${ }^{[16]}$.

74 The respective PSA1 pattern has been widely recognised as being highly correlated with ENSO, where previous studies argue that it results in part as an atmospheric response to ENSO [15, 17, 18]. While regression analysis shows that there is indeed a very close relationship between PSA1 and

77 ENSO (Fig. 1a), the origin of this relationship remains an active area of research ${ }^{[19,20]}$. Meanwhile, 78 the connection between the atmospheric PSA2 and tropical SST variability is less clear. Ref [15] 79 argues that PSA2 is responsible for the quasi-biennial component of ENSO (Fig. 1b). Although ENSO-PSA correlations are widely documented, the processes by which ENSO and the PSA mode 81 are dynamically connected, and, in particular, how the PSA mode feeds back to the tropics, remain 82 unclear.

83 Diverse views exist on the mechanisms that link the atmospheric PSA mode with ENSO. Some 84 studies ${ }^{[15,17,21,22]}$ argue that the ENSO-PSA connection is directly linked via atmospheric Rossby 85 wave propagation. However, other studies ${ }^{[20,23,24]}$ point out that synoptic scale Rossby waves are 86 primarily generated and trapped locally within the Southern Hemisphere subtropical and polar jet 
87 streams. Unlike the teleconnection between the Madden-Julian oscillation and North Atlantic

88 oscillation $^{[25]}$, there is little direct dynamical evidence in terms of Rossby wave source or wave

89 activity flux (see, for example, refs ${ }^{[19,20,26]}$ ) to suggest that poleward propagating large-scale

90 tropical Pacific Rossby waves, potentially initiated by ENSO variability, teleconnect the PSA to

91 ENSO. Even if sufficient Rossby wave sources were to be generated in the equatorial Pacific, ray

92 tracing theory and wave activity flux calculations make it readily apparent that they are blocked

93 by a reflecting barrier associated with the presence of the subtropical jet, and low latitude wave

94 breaking, from propagation to the midlatitudes. Instead, Ref ${ }^{[27]}$ argues that the PSA mode is linked

95 to ENSO via a direct modulation of the midlatitude jets by the thermal winds generated by tropical

convection that is highly correlated with ENSO. This indirectly influences the interannual

97 variability of coherent synoptic features forming within these midlatitude waveguides where local

98 Rossby waves sources are prevalent.

\section{Oceanic signal driven by the PSA mode}

101 Analysis of South Pacific SST shows that the first two modes of SST variability, referred to as the 102 SPDO ${ }^{[3,4]}$ and South Pacific quadrupole SST pattern ${ }^{[8]}$, are primarily driven by the variability 103 associated with the atmospheric PSA1 and PSA2 patterns (Figs. $1 \mathrm{e}$ and $\mathrm{f}$ ). Using a univariate first104 order autoregressive (AR1) model ${ }^{[28,29]}$, we found that the integrated atmospheric PSA1 and PSA2 105 variability could explain a significant fraction of the variance of the first two SST modes in the 106 South Pacific Ocean (Figs. 1 e and f; R=0.76 and 0.71, respectively; significant at $>99 \%$ level; see 107 the methods section for details). 
A second integration of the AR1 model (following a similar approach to refs ${ }^{[4,30]}$ ) - with the first integration being from the atmosphere to the surface ocean, and the second integration from the surface ocean to the subsurface ocean - reveals that the leading SST mode is further reddened by the extratropical upper ocean (Fig. 1 e). The integrated SPDO signal (blue curve in Fig. 1 e) resembles the time evolution of the leading vertically averaged temperature (VAT) mode through the upper $300 \mathrm{~m}$ of the ocean (see the methods section), with a temporal correlation of $\mathrm{R}=0.88$ (statistically significant at the 99\% level). To first order, we conclude that the South Pacific Ocean integrates the fast-varying atmospheric "noise" forcing to produce a "reddened" oceanic signal, with a pronounced increase in amplitude of the spectrum at low frequencies and decreased amplitude at high frequencies, where the main potentially predictable signal is generated on the time scales of the ocean dynamics.

\section{Oceanic noise driven by the PSA mode}

The extratropical South Pacific not only responds via reddening processes to the fast-varying atmosphere but also via coherence resonances ${ }^{[31]}$. Specifically, the forcing due to coherent synoptic scale disturbances in the atmosphere associated with the PSA imprints onto the surface ocean further enhancing internal SST variability at its preferred frequency. To examine this mechanism, we generalised the univariate AR1 model to a higher-dimensional multivariate field through inclusion of SST anomalies in the tropical and South Pacific oceans using a linear inverse model ${ }^{[32]}$ (LIM; see methods section). Previous studies have shown that the tropical and South Pacific oceans can be approximated as a stochastically forced linear system, where different dynamical processes are represented by distinct damping time scales via the decomposition of the $\operatorname{LIM}^{[5,32-35]}$. 
131 Fig. 2 shows the fastest damped SST mode pair with a damping time scale of two months. This

132 pair of complex patterns (Figs. $2 \mathrm{a}$ and b) constitutes a single propagating wave train with a

133 complete cycle of 26 months. Along with the propagating features, the spatial patterns of the fastest

134 damped modes also bear a strong resemblance to the atmospheric PSA1 and PSA2 patterns.

135 Projecting the corresponding time series of the real and imaginary components of the fastest

136 damped mode onto the monthly 500-hPa geopotential height (Z500) anomalies produces

137 regression maps (Figs. $2 \mathrm{c}$ and d) that closely resemble the atmospheric PSA1 and PSA2 with

138 spatial correlations of 0.74 and 0.69 (statistically significant at the $95 \%$ level), respectively, in the

139 South Pacific region. This suggests that the high-frequency atmospheric PSA fluctuations can

140 excite SST modes whose frequencies are subject to the atmospheric drivers. In contrast to the

141 reddening processes that act to excite the potentially predictable low frequency ocean signal, the

142 pair of most damped SST modes are correlated with the atmospheric PSA forcing that acts as a

143 stochastic forcing on the SST system.

\section{The optimal growth and extratropical precursor}

146 Previous studies have extensively discussed the tropical and extratropical precursors of ENSO ${ }^{[8,}$

$14710,32,36,37]$. Despite there being little direct evidence, in terms of atmospheric dynamics, to show

148 that the atmospheric PSA mode can directly modulate ENSO evolution in the tropics ${ }^{[27]}$, the

149 indirect influence has nevertheless been widely identified in both observations ${ }^{[11]}$ and model

150 simulations ${ }^{[36,38]}$ via the "atmosphere $\rightarrow$ extratropical ocean $\rightarrow$ tropical ocean" pathway. One way

151 in which this indirect influence may occur is through a "seasonal footprinting" mechanism [39],

152 where the atmospheric forcing drives an extratropical anomalous SST "footprint" in the boreal 
153 spring, which persists through the boreal summer, and sustains wind stress anomalies in the tropics 154 that are crucial to initiate ENSO events.

155 The LIM approach enables an objective determination of the optimal initial perturbations that 156 maximise, for example, ENSO and SPDO growth. This provides an ideal framework through 157 which to investigate the dynamical precursors and predictability of the peak phases of ENSO and 158 the SPDO. Unlike lead-lag correlations that have been widely applied to identify ENSO precursors $159[8,9,11]$, the LIM represents a multivariate linear stochastically forced model and provides a 160 dynamical approximation (see methods section) that satisfies conditionally causal ${ }^{[40]}$ relationships 161 between the optimal precursors and their peak phases.

162 The interactions between the non-normal damping SST modes can give rise to a transient 163 amplification of the variance of the deterministic SST system at a preferred temporal growth scale. 164 Such transient amplification is useful in sampling and interpreting errors in initial conditions ${ }^{[41]}$ 165 and explains the actual variance growth in the system ${ }^{[32]}$. Transient amplification of monthly 166 tropical and South Pacific SST anomalies allows a specific set of initial perturbations to develop 167 into the peak phases that are found to exist at 6- to 10 -month lead times ${ }^{[5,9,32,33,42]}$. Our LIM 168 experimental results show that the optimal growth time of SST anomalies in the tropical and South 169 Pacific oceans (see methods section) is 9 months. These results imply the existence of linear 170 growth events that act to maximise ENSO and SPDO development leading to enhanced 171 predictability if the initial perturbations can be sufficiently well specified.

172 The spatial patterns of the initial SST perturbations in the tropical Pacific and South Pacific are 173 shown in Figs. 3 a and c, respectively. These initial patterns co-evolve over nine months into the 174 optimal final peak phases in Figs. $3 \mathrm{~b}$ and d, which are closely associated with ENSO and the 175 SPDO with pattern correlations of 0.99 and 0.97 (statistically significant at the $99 \%$ level), 


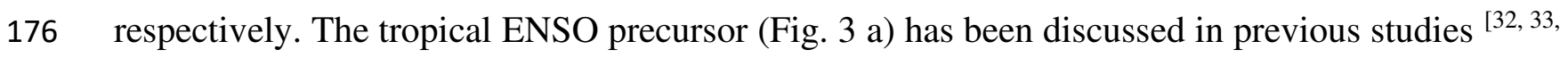

$177{ }^{37]}$ and may be associated with the recharge-discharge mechanism as first described by Jin ${ }^{[43]}$. The

178 extratropical precursor (Fig. 3 c) resembles a quadrupole structure similar to the second South

179 Pacific SST mode with the pattern correlation of 0.85 (significant at the $95 \%$ level). The initial

180 and final spatial patterns (Figs. $3 \mathrm{c}$ and d) were projected onto the monthly SST anomalies to

181 reconstruct the time series. The reconstructed time series of the optimal initial perturbations and

182 final peak phases in the South Pacific (Figs. 3 e and f) are highly correlated with the second and

183 first South Pacific SST modes with temporal correlations of 0.86 and 0.98 (statistically significant

184 at the $99 \%$ level), respectively. This suggests that the South Pacific quadrupole SST pattern, which

185 is primarily driven by the atmospheric PSA2, is the optimal local (linear) precursor that maximises

186 the SPDO growth. Given the almost synchronous manifestation of feature relationships between

187 ENSO and the SPDO ${ }^{[3,4]}$, these results imply that the extratropical SST precursor of ENSO is also

188 related to the quadrupole SST pattern in the South Pacific Ocean. Our results support the "seasonal 189 footprinting" mechanism whereby the atmospheric PSA2 excites the South Pacific quadrupole 190 SST anomalies, which then persist through the boreal summer to guide ENSO growth.

\section{A new paradigm for South Pacific climate variability and predictability}

Fig. 4 summarises the atmospheric PSA mode and its South Pacific Ocean responses based on the combined results from the AR1 and LIM investigations. Our results suggest that the eastwardpropagating PSA mode provides an important source of atmospheric forcing to excite the extratropical South Pacific Ocean responses operating on multiple time scales via reddening

197 processes and coherence resonances. To first order, the close relationships between the integrated PSA1 and PSA2 patterns and the leading South Pacific SST modes support the general concept 
199 that fast atmospheric variations are a critically important excitation source of low frequency 200 oceanic variability. The leading integrated subsurface temperature mode, that resembles the spatial 201 pattern of its SST counterpart, can be regarded as a cumulative response to atmospheric PSA1 202 forcing. That is, the inclusion of extratropical subsurface processes further reddens the SST 203 variations thereby enhancing the most persistent and potentially predictable signal. Furthermore, 204 the atmospheric PSA1 and PSA2 patterns can excite SST variations via coherence resonances such 205 that the resultant fastest damping SST modes are slaves to the atmospheric forcing but 206 synchronised to the spatiotemporal features of the real and imaginary components of the 207 propagating PSA.

208 The extratropical SST precursor of ENSO and SPDO growth is strongly associated with the South 209 Pacific quadrupole SST pattern. The set of initial conditions related to this quadrupole pattern can 210 optimally determine how the SST anomalies will evolve along its deterministic (linear) trajectory 211 and lead to the SPDO and ENSO peaks over the following nine months, and from which the linear 212 predictability intrinsic to the SST system can be inferred. While the influence of the atmospheric 213 PSA mode on tropical SST variability is less apparent than the influence of the tropical SST 214 variability on the PSA mode ${ }^{[8,20,27]}$, our results indicate that the South Pacific quadrupole SST 215 pattern acts as an oceanic bridge that links the atmospheric PSA forcing to ENSO dynamics centred 216 in the tropics, and represents the most probable pathway for midlatitude atmospheric variability to 217 influence tropical SST via an indirect "seasonal footprinting" mechanism. Although the actual 218 observed nonlinear dynamics are more complicated than the tangent linear dynamics that form the 219 basis of the LIM propagator, the essential dynamics of the system are captured. The proposed 220 paradigm provides a new mechanistic framework to understand the dynamics and predictability of 
221 South Pacific climate variability and to interpret extratropical atmospheric drivers and the oceanic

222 responses on multiple time scales.
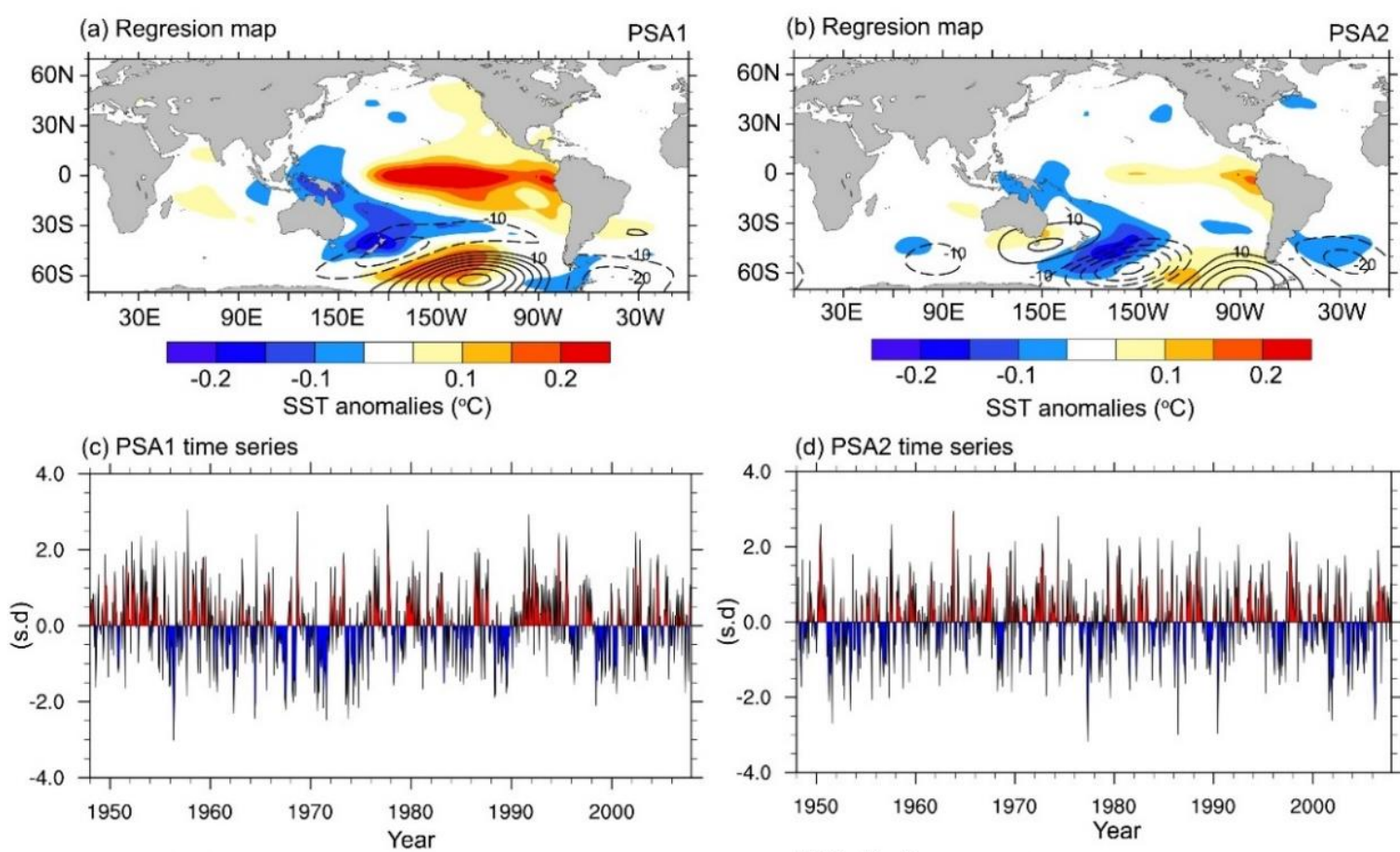

(d) PSA2 time series
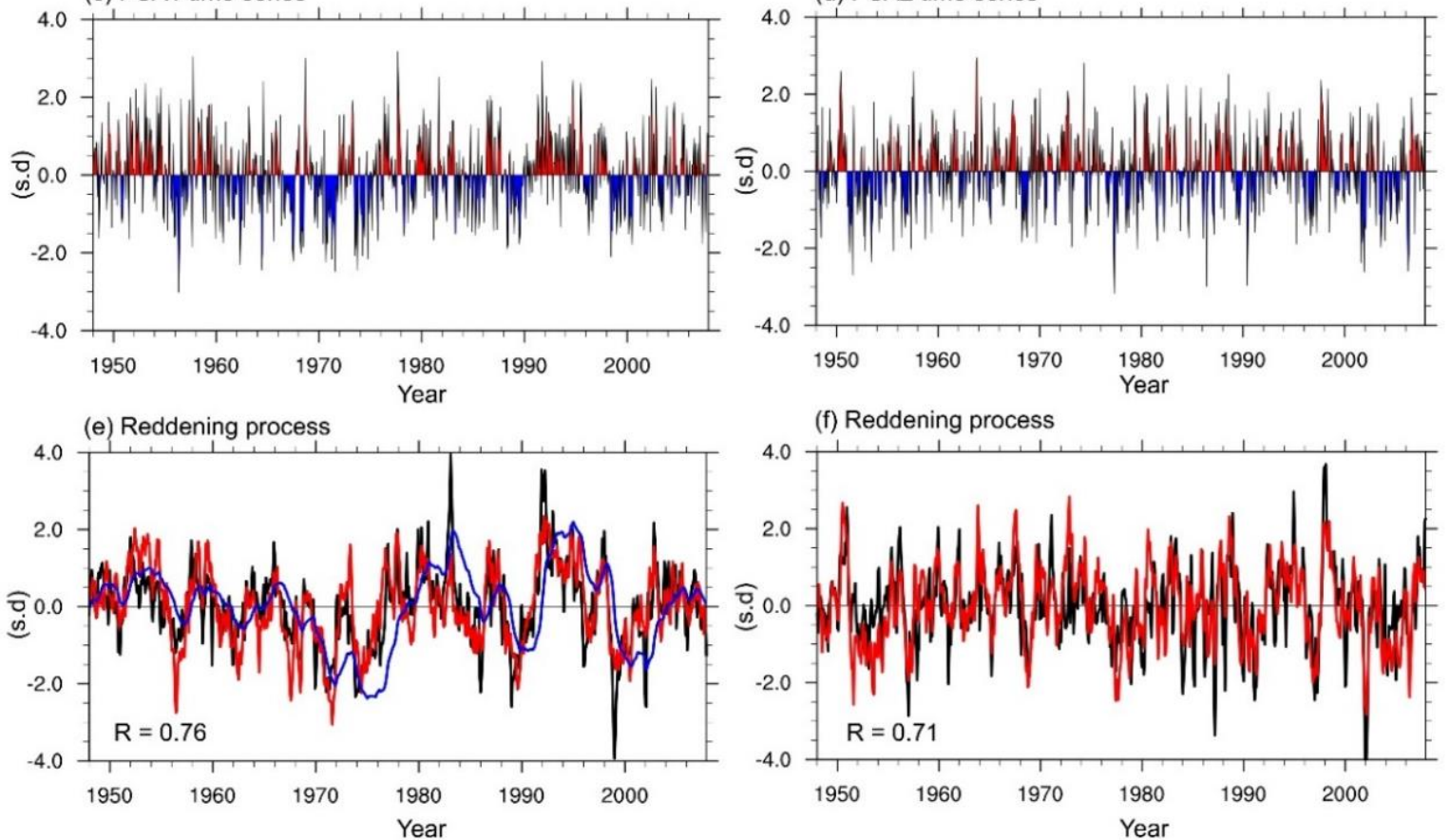

Fig. 1 | Atmospheric forcing of the leading two SST modes in the South Pacific Ocean. a, b, anomalies (NCEP-NCAR; contour) and monthly SST anomalies (ACCESS-O; shade) onto the

227 PSA1 and PSA2 time series, respectively. (c) and (d), The corresponding normalised PSA1 and 228 PSA2 time series. (e) and (f), The time series of the leading two SST modes of variability in the 229 South Pacific Ocean (ACCESS-O; black) are reconstructed (red) using an AR1 model forced by 
the PSA1 and PSA2 time series. The blue curve in (e) indicates the second integration forced by

231 the SST SPDO time series. The units are in standard deviations (s.d.). The significance of the

232 temporal correlations is estimated taking account of the effective number of degrees of freedom

233 due to serial correlation ${ }^{[44]}$.
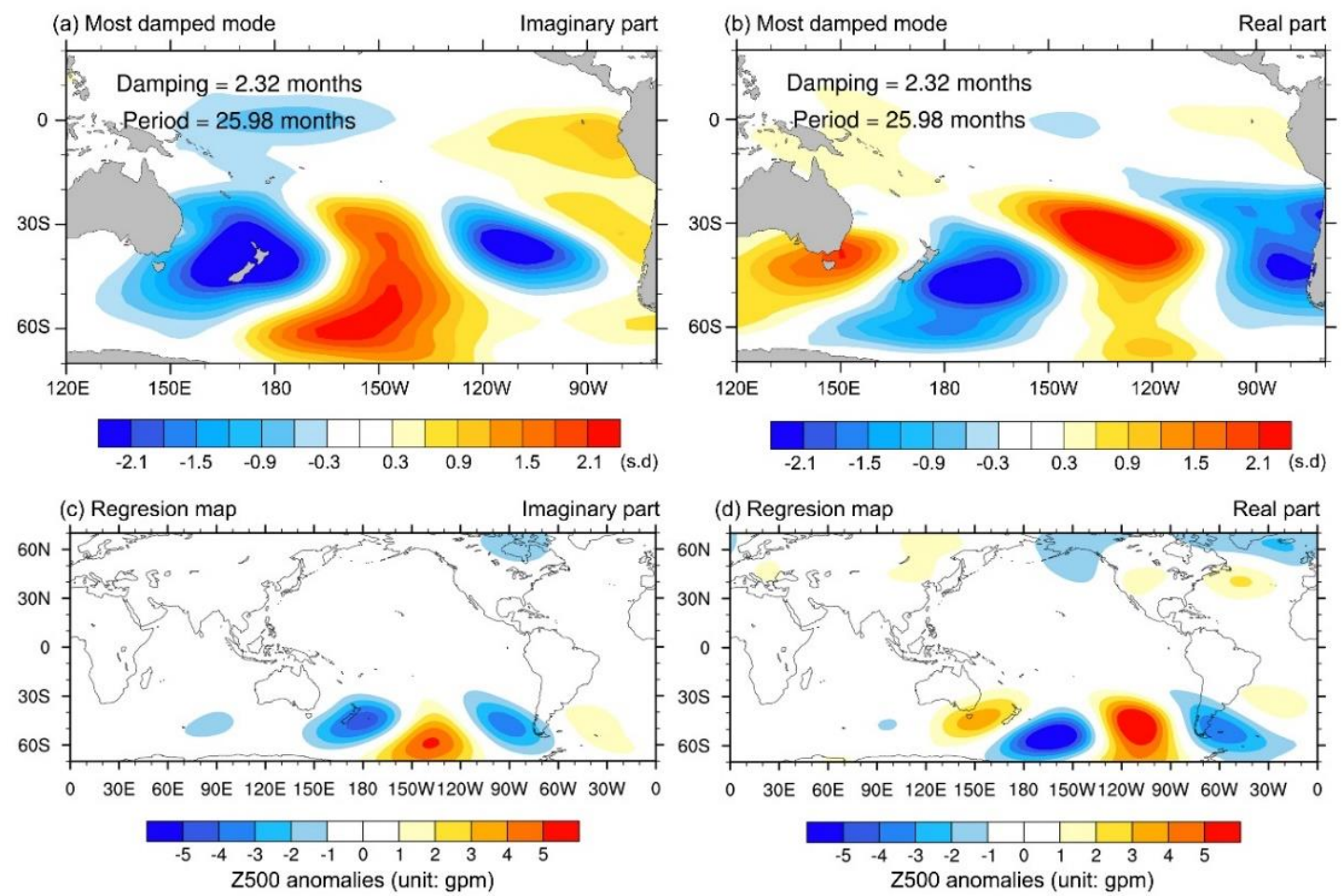

234

Fig. 2 The fastest damped SST modes. a, b, The imaginary (a) and real (b) parts of the fastest damped SST modes are obtained using a reduced-order linear inverse model (see the text for details). The pair of SST modes shown has the fastest damping time scale of 2.3 months. $\mathbf{c}$, d, the maps are obtained by regressing the monthly Z500 anomalies (NCEP-NCAR) onto the time series of the imaginary (c) and real (d) parts of the fastest damped modes. 

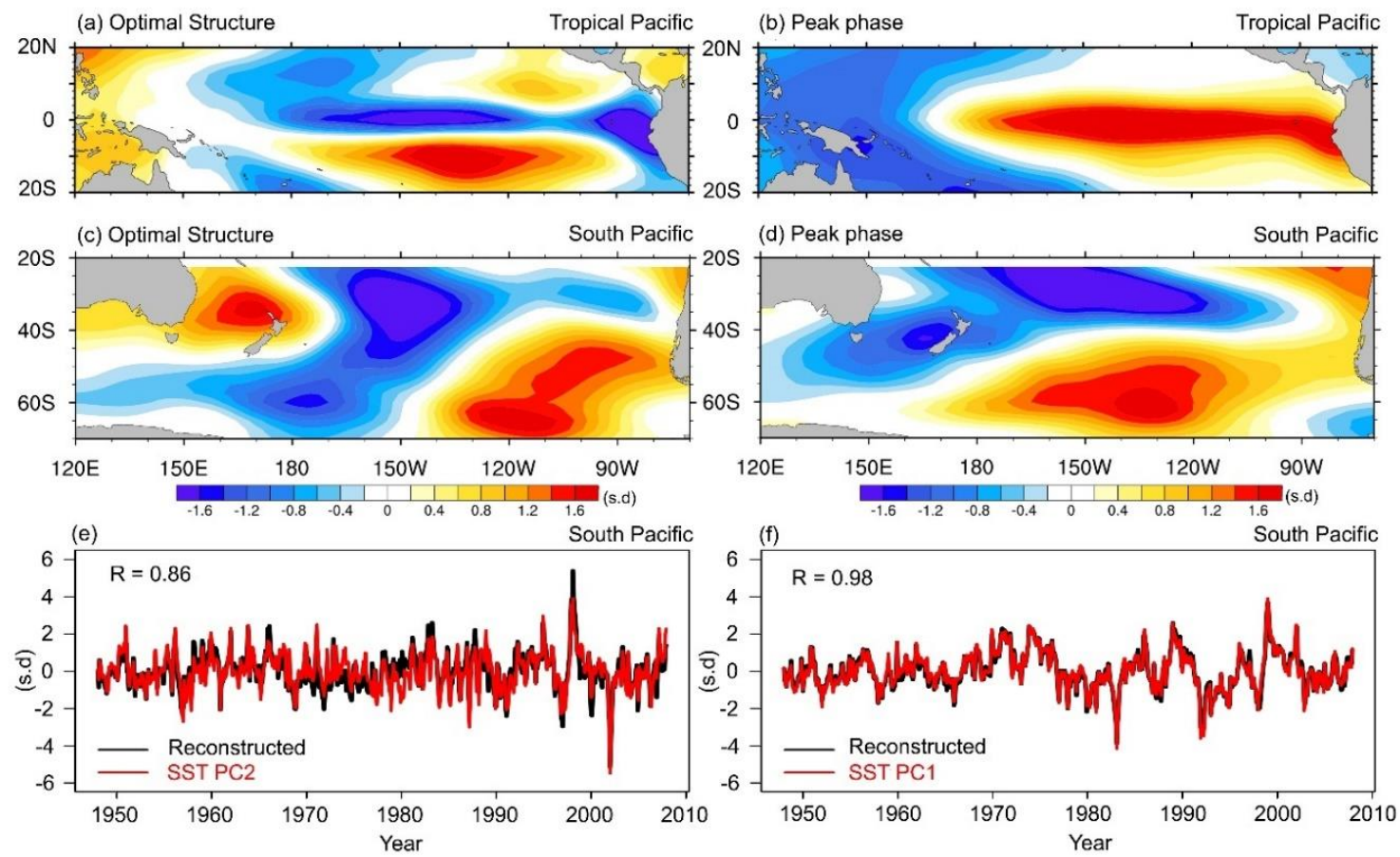

243 Fig. 3 | The optimal evolution of the tropical and South Pacific SST. The optimal initial

244 structures in the (a) tropical Pacific and (c) South Pacific are obtained using a linear inverse model

245 (see the text for details), which linearly co-evolve over 9 months into their peak phases in the (b)

246 tropical Pacific and (d) South Pacific. The spatial patterns shown in a-d are normalised according

247 to the variance in each domain. e and $\mathbf{f}$, the reconstructed time series of the (e) optimal initial

248 structure and (f) peak phase in the South Pacific (black) are compared with the time series of the

249 leading two South Pacific SST modes (red). 


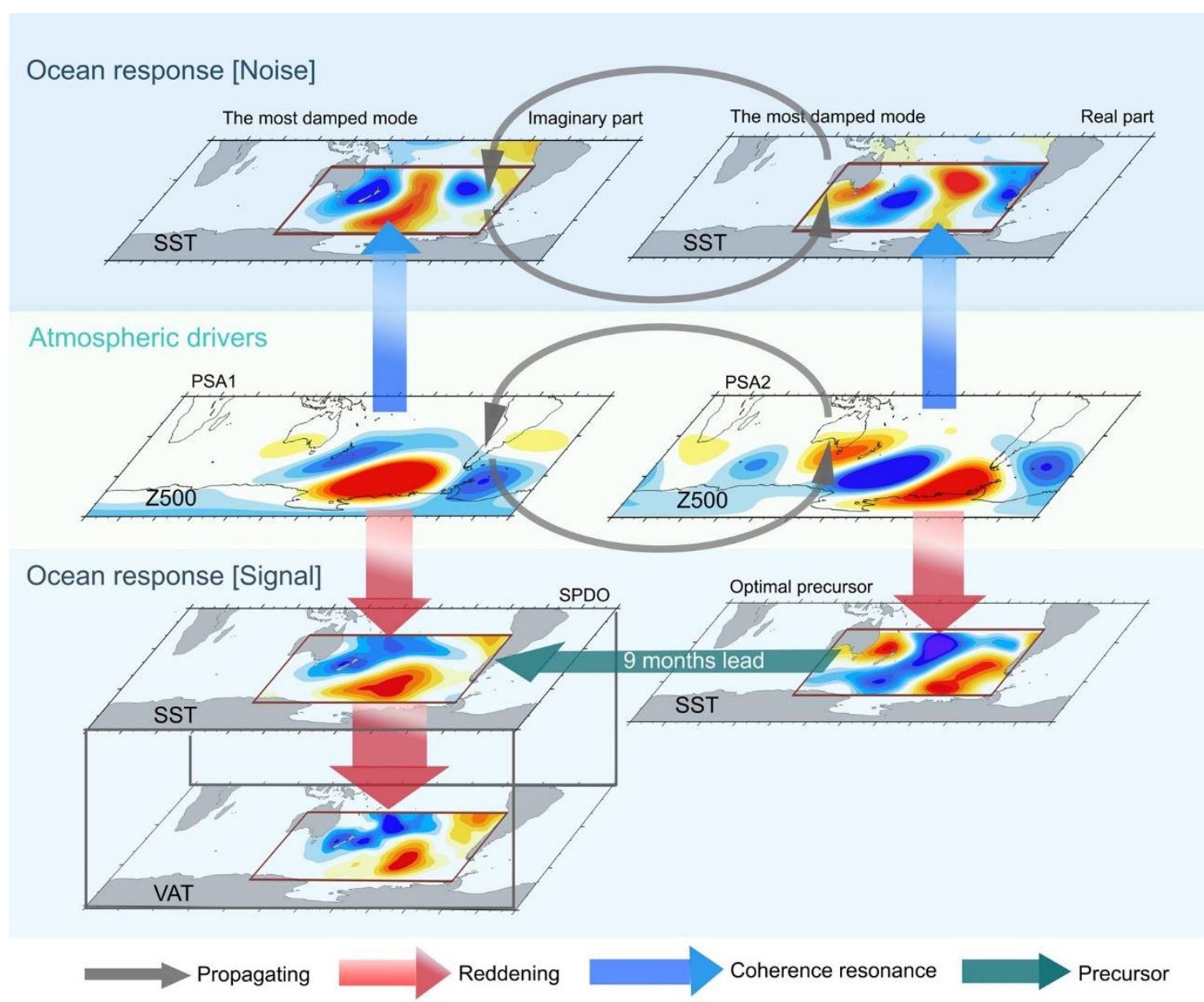

Fig. 4 | Schematic of new paradigm for South Pacific Ocean variability and predictability.

252 The top panels are the same as shown in Figs. $2 \mathbf{a}$ and $\mathbf{b}$ indicating the fastest damped mode pair.

253 The middle panels are the second and third modes of the monthly Z500 anomalies (NCEP-NCAR)

254 in the southern hemisphere. The left panels of the bottom layer are the leading EOF modes of the

255 monthly SST anomalies and VAT anomalies in the South Pacific Ocean, respectively. The right

256 panel of the bottom layer is the same as shown in Fig. $3 \mathbf{c}$ indicating the optimal precursor of the

257 SPDO (illustrated by the green arrow). Red arrows indicate reddening processes. Blue arrows

258 indicate coherence resonances. Grey arrows indicate the propagating features of the corresponding

259 modes. 


\section{References}

261 1. Mantua, N. J. \& Hare, S. R. The Pacific decadal oscillation. J. Oceanogr. 58, 35-44 (2002).

262 2. Power, S. et al. Inter-decadal modulation of the impact of ENSO on Australia. Clim. Dynam. 15, 319-324 (1999).

3. Chen, X. \& Wallace J. M. ENSO-like variability: 1900-2013*. J. Climate 28, 9623-9641(2015).

4. Lou, J., Holbrook, N. J. \& O'Kane T. J. South Pacific decadal climate variability and potential predictability. J. Climate 32, 6051-6069 (2019).

5. Lou, J., O'Kane, T. J. \& Holbrook, N. J. A linear inverse model of tropical and South Pacific seasonal predictability. J. Climate 33, 4537-4554 (2020).

6. Taschetto, A.S. et al. ENSO atmospheric teleconnections, El Niño Southern Oscillation in a Changing Climate 309-335 (2020).

7. Sprintall, J. et al. ENSO oceanic teleconnections, El Niño Southern Oscillation in a Changing Climate 337-359 (2020).

8. Ding, R., Li, J. \& Tseng, Y.-h. The impact of South Pacific extratropical forcing on ENSO and comparisons with the North Pacific. Clim. Dynam. 44, 2017-2034 (2015).

9. Zhao, Y. \& Di Lorenzo, E. The impacts of extra-tropical ENSO precursors on tropical Pacific decadal-scale variability. Sci. Rep. 10, 3031 (2020).

10. Zhang, H., Clement, A. \& Di Nezio, P. The South Pacific meridional mode: a mechanism for ENSO-like variability. J. Climate 27, 769-783 (2014).

11. You, Y. \& Furtado, J. C. The role of South Pacific atmospheric variability in the development of different types of ENSO. Geophys. Res. Lett. 44, 7438-7446 (2017).

12. Lau, K.M., Sheu, P. J. \& Kang, I.S. Multiscale low-frequency circulation modes in the global atmosphere. J. Atmos. Sci. 51, 1169-1193 (1994).

13. Echevarria, E.R. et al. Influence of the Pacific-South American modes on the global spectral wind-wave climate. J. Geophys. Res. Oceans 125, e2020JC016354 (2020).

14. Irving, D. \& Simmonds, I. A new method for identifying the Pacific-South American pattern and its influence on regional climate variability. J. Climate 29, 6109-6125 (2016).

15. Mo, K.C. \& Paegle, J. N. The Pacific-South American modes and their downstream effects. Int. J. Climatol. 21, 1211-1229 (2001).

16. Rehbein, A. et al. Severe weather events over southeastern Brazil during the 2016 dry season. Adv. Meteorol. 2018, 4878503 (2018).

17. Karoly, D. J. Southern Hemisphere circulation features associated with El Niño-Southern Oscillation events. J. Climate 2, 1239-1252 (1989).

18. Cai, W. et al. Climate impacts of the El Niño-Southern Oscillation on South America. Nat. Rev. Earth Environ. 1, 215-231(2020).

19. Karoly, D. J., Plumb, R.A. \& Ting, M. Examples of the horizontal propagation of quasi-stationary waves. J. Atmos. Sci. 46, 2802-2811(1988).

20. Li, X. et al. A Rossby wave bridge from the tropical Atlantic to west Antarctica. J. Climate 28, 2256-2273 (2015).

21. Renwick, J. A. \& Revell, M. J. Blocking over the South Pacific and Rossby wave propagation. Mon. Weather Rev. 127, 2233-2247 (1999).

22. Cai, W. et al. Teleconnection pathways of ENSO and the IOD and the mechanisms for impacts on Australian rainfall. J. Climate 24, 3910-3923 (2011).

23. Ambrizzi, T. \& Hoskins, B.J. Stationary rossby-wave propagation in a baroclinic atmosphere. $Q$. J. R. Meteorol. Soc. 123, 919-928 (1997).

24. Ambrizzi, T., Hoskins, B.J. \& Hsu, H.-H. Rossby wave propagation and teleconnection patterns in the austral winter. J. Atmos. Sci. 52, 3661-3672 (1995).

25. Lin, H. \& Brunet, G. Extratropical response to the MJO: nonlinearity and sensitivity to the initial state. J. Atmos. Sci. 75, 219-234 (2018). 
26. O'Kane, T. J. et al. On the dynamics of persistent states and their secular trends in the waveguides of the Southern Hemisphere troposphere. Clim. Dynam. 46, 3567-3597 (2015).

27. O'Kane, T. J., Monselesan, D. P. \& Risbey, J. S. A multiscale reexamination of the Pacific-South American pattern. Mon. Weather Rev. 145, 379-402 (2017).

28. Frankignoul, C. \& Hasselmann, K. Stochastic climate models, Part II Application to sea-surface temperature anomalies and thermocline variability. Tellus 29, 289-305 (1977).

29. Hasselmann, K. Stochastic climate models Part I. Theory. Tellus 28, 473-485 (1976).

30. Di Lorenzo, E. \& Ohman, M. D. A double-integration hypothesis to explain ocean ecosystem response to climate forcing. Proc. Natl. Acad. Sci. U.S.A. 110, 2496-9 (2013).

31. Pierini, S. Low-frequency variability, coherence resonance, and phase selection in a low-order model of the wind-driven ocean circulation. J. Phys. Oceanogr. 41, 1585-1604 (2011).

32. Penland, C. \& Sardeshmukh, P. D. The optimal growth of tropical sea surface temperature anomalies. J. Climate 8,1999-2024 (1995).

33. Capotondi, A. \& Sardeshmukh, P. D. Optimal precursors of different types of ENSO events. Geophys. Res. Lett. 42, 9952-9960 (2015).

34. Newman, M. Interannual to decadal predictability of tropical and North Pacific sea surface temperatures. J. Climate, 20, 2333-2356 (2007).

35. Penland, C. \& Magorian, T. Prediction of Niño 3 sea surface temperatures using linear inverse modeling. J. Climate 6, 1067-1076 (1993).

36. Liguori, G. \& Di Lorenzo, E. Separating the North and South Pacific meridional modes contributions to ENSO and tropical decadal variability. Geophys. Res. Lett. 46, 906-915 (2019).

37. Penland, C. \& Matrosova, L. Studies of El Niño and interdecadal variability in tropical sea surface temperatures using a nonnormal filter. J. Climate 19, 5796-5815 (2006).

38. Zhang, H. et al. Equatorial signatures of the Pacific meridional modes: Dependence on mean climate state. Geophys. Res. Lett. 41, 568-574 (2014).

39. Vimont, D. J., Wallace, J.M. \& Battisti, D. S. The seasonal footprinting mechanism in the Pacific: Implications for ENSO. J. Climate 16, 2668-2675 (2003).

40. Granger, C. W. J. Some recent development in a concept of causality. J. Econom. 39, 199-211 (1988).

41. Moore, A. M. \& Kleeman, R. The dynamics of error growth and predictability in a coupled model of ENSO. Q. J. R. Meteorol. Soc. 122, 1405-1446 (1996).

42. Newman, M., Alexander, M. A. \& Scott, J. D. An empirical model of tropical ocean dynamics. Clim. Dynam. 37, 1823-1841(2011).

43. Jin, F.-F. An equatorial ocean recharge paradigm for ENSO. Part I: Conceptual model. J. Atmos. Sci. 54, 811-829 (1997).

44. Davis, R.E. Predictability of sea surface temperature and sea level pressure anomalies over the North Pacific Ocean. J. Phys. Oceanogr. 6, 249-266 (1976).

45. Kalnay, E. et al. The NCEP/NCAR 40-year reanalysis project. Bull. Am. Meteorol. Soc. 77, 437472 (1996).

46. O'Kane, T. J. et al. Storm tracks in the Southern Hemisphere subtropical oceans. J. Geophys. Res. Oceans 119, 6078-6100 (2014).

47. von Storch, H. et al. Principal oscillation patterns: A review. J. Climate 8, 377-400 (1995).

48. Lou, J., O'Kane, T.J. \& Holbrook, N. J. A linear inverse model of tropical and South Pacific climate variability: Optimal structure and stochastic forcing. J. Climate 34, 143-155 (2021). 


\section{Methods}

358 The reanalysis data used here were monthly detrended anomalies of the 500-hPa geopotential 359 heights (Z500) from the National Centers for Environmental Prediction/National Center for 360 Atmospheric Research (NCEP-NCAR) ${ }^{[45]}$. The monthly detrended sea surface temperature (SST)

361 anomalies were generated using an atmosphere-forced ocean model, the Australian Community 362 Climate and Earth-System Simulator-Ocean (ACCESS-O). The model configuration is described 363 in ref ${ }^{[46]}$. The original $360 \times 300$ tripolar ACCESS-O model grid has been remapped to a regular $3642.5^{\circ} \times 2.5^{\circ}$ grid. The reanalysis data and model simulations were linearly detrended at each grid 365 point, with monthly anomalies calculated by removing the climatological monthly mean.

366 The atmospheric PSA1 and PSA2 patterns were defined by computing the second and third 367 empirical orthogonal functions/principal components (EOFs/PCs) of the monthly Z500 anomalies 368 in the Southern Hemisphere, respectively. The leading two South Pacific SST modes, which are 369 referred to as the South Pacific decadal oscillation (SPDO) and the South Pacific quadrupole SST 370 pattern, were obtained by computing the first and second EOFs/PCs of the monthly SST anomalies 371 in the South Pacific Ocean domain from $\left(20^{\circ} \mathrm{S}-70^{\circ} \mathrm{S} ; 120^{\circ} \mathrm{E}-60^{\circ} \mathrm{W}\right)$. The subsurface temperature 372 variability was characterised by spatiotemporal variations of vertically averaged temperatures 373 (VAT) through the upper 300-m of the ocean, that includes the mixed layer and thermocline across 374 most regions. The subsurface SPDO is derived from the first EOF/PC of the VAT in the South 375 Pacific Ocean $\left(20^{\circ} \mathrm{S}-70^{\circ} \mathrm{S} ; 120^{\circ} \mathrm{E}-60^{\circ} \mathrm{W}\right)$. Statistical significance tests of the correlation 376 coefficients calculated in this study took account of the effective number of degrees of freedom 377 due to serial correlation in the time series, following the method of ref ${ }^{[44]}$. 
378 The oceanic reddening response to atmospheric PSA forcing was quantified using a doubly

379 integrated first-order autoregressive (AR1) model. A univariate AR1 model can be written as

$$
\frac{d x}{d t}=l x+\xi
$$

381 where $x$ is a time series and $-1 / l$ is associated with the damping time scale. This univariate AR1 382 process only has one degree of freedom and is unable to oscillate when the damping coefficient $l$ 383 is positive ${ }^{[47]}$. Predictability of the time series $x$ is largely limited by the damping scale $-1 / l$. $\xi$ 384 in Eq. (1) represents a high-frequency noise forcing term.

385 The optimal damping time scales are 6 months and 5 months, respectively, when the time series 386 of the PSA1 and PSA2 patterns were specified as the atmospheric forcing of the first two South 387 Pacific SST modes in the first AR1 integration from the atmosphere to the surface ocean. In the 388 second integration from the surface ocean to the subsurface ocean, the optimal damping time scale 389 is 15 months when the time series of the SST SPDO was specified as the forcing of the leading 390 South Pacific VAT mode.

391 The pair of fastest damped noise mode and optimal initial perturbations were estimated using a 392 generalised AR1 model, here referred to as the linear inverse model (LIM) ${ }^{[32]}$, or alternatively 393 termed principal oscillation pattern (POP) analysis ${ }^{[47]}$. The LIM can be written in the form of a 394 linear stochastic differential equation:

$$
\frac{\mathrm{dX}}{\mathrm{dt}}=\mathbf{L X}+\boldsymbol{\xi}
$$
where the evolution of the state vector $\mathbf{X}$ can be expressed as the sum of the deterministic dynamics, 397 LX, that constitute "slow" processes and the stochastic forcing term, $\xi$, that constitutes "fast" 398 processes. In this study, we defined the model state vector $\mathbf{X}$ as 


$$
\mathbf{X}=\left[\begin{array}{l}
\mathbf{S S T}_{\mathbf{T P}} \\
\mathbf{S S T}_{\mathbf{S P}}
\end{array}\right]
$$

400 where we considered monthly SST anomalies from the tropical Pacific (i.e., $\mathbf{S S T}_{\mathbf{T P}}$ in the region $40120^{\circ} \mathrm{S}-20^{\circ} \mathrm{N}, 120^{\circ} \mathrm{E}-60^{\circ} \mathrm{W}$ ) and South Pacific Ocean (i.e., $\mathbf{S S T}_{\mathbf{S P}}$ in the region $22.5^{\circ} \mathrm{S}-70^{\circ} \mathrm{S}, 120^{\circ} \mathrm{E}-$ $\left.40260^{\circ} \mathrm{W}\right)$. We retained the leading 4 tropical Pacific and 6 South Pacific SST EOFs/PCs in the LIM 403 to reduce the spatial degrees of freedom and ensure linearity and stability of the SST system. The 404 robustness of the LIM has been demonstrated in the refs ${ }^{[5,48]}$, showing that the choices of using 405 the leading 10 to 16 EOFs/PCs to construct the state vector $\mathbf{X}$ and the different combinations of 406 the leading EOFs/PCs used from the different regions give similar results.

407 The dynamical operator $\mathbf{L}$ in Eq. (2) encapsulates the evolution of the state vector $\mathbf{X}$ and can be 408 estimated from

$$
\mathbf{L}=\tau_{0}^{-1} \ln \left[\mathbf{C}\left(\tau_{0}\right) \mathbf{C}(0)^{-1}\right]
$$

410 where $\mathbf{C}\left(\tau_{0}\right)$ and $\mathbf{C}(0)$ are the time-lagged and zero-lagged (cross-)covariance matrices of the state

411 vector $\mathbf{X}$. That is, $\mathbf{C}\left(\tau_{0}\right)=\left\langle\mathbf{X}\left(t+\tau_{0}\right) \mathbf{X}^{\mathbf{T}}(t)\right\rangle$ and $\mathbf{C}(0)=\left\langle\mathbf{X}(t) \mathbf{X}^{\mathbf{T}}(t)\right\rangle$, where the angle brackets 412 here denote an ensemble average or a time average over all $t$ for variables with stationary statistics.

413 Here, $\tau_{0}=1$ month was used to ensure the stability of the LIM. Previous studies ${ }^{[5,48]}$ examined 414 the sensitivity of using different choices of $\tau_{0}$ to estimate the dynamical operator $\mathbf{L}$, finding that 415 the LIM is insensitive to $\tau_{0}$ ranging from 1 month to 6 months.

416 For a system described by Eq. (2), the most probable evolution at time $t+\tau$ can be determined by

$$
\mathbf{X}(t+\tau)=\mathbf{G}(\tau) \mathbf{X}(t)=e^{\mathbf{L} \tau} \mathbf{X}(t)
$$

418 given a state vector $\mathbf{X}(t)$. 
419 The damping modes can then be diagnosed by applying eigen-decomposition to the dynamical 420 operator $\mathbf{L}$ (i.e., $\mathbf{L p}=\lambda \mathbf{p}$ ). Since the $\mathbf{L}$ matrix is not symmetric, some or all of its eigenvalues $\lambda$ 421 and eigenvectors $\mathbf{p}$ are complex. The damping time scales $-1 / \sigma$ and/or the oscillatory periods $2 \pi / \omega$ 422 of the damping modes can be directly estimated from the corresponding eigenvalues $\lambda=\sigma+i \omega$, 423 where $\sigma$ and $\omega$ are the real and imaginary parts of $\lambda$, respectively. In this study, the pair of complex 424 damping modes that has the fastest damping time scale has been discussed.

425 The transient growth over any time interval $\tau$ can be written as the norm of the final state $\mathbf{X}(t)$ 426 divided by the norm of the initial state $\mathbf{X}(0)$ :

$$
\gamma^{2}=\frac{\|\mathbf{X}(t)\|^{2}}{\|\mathbf{X}(0)\|^{2}}=\frac{\mathbf{X}^{\mathrm{T}}(t) \mathbf{X}(t)}{\mathbf{X}^{\mathrm{T}}(0) \mathbf{X}(0)}=\frac{\mathbf{X}^{\mathrm{T}}(0) \mathbf{G}^{\mathrm{T}}(\tau) \mathbf{G}(\tau) \mathbf{X}(0)}{\mathbf{X}^{\mathrm{T}}(0) \mathbf{X}(0)}
$$

428 The optimal growth that maximises the amplification of the variance can be estimated as the 429 leading eigenvalue $\gamma_{1}^{2}$ of $\mathbf{G}^{\mathrm{T}}(\tau) \mathbf{G}(\tau)$. The leading eigenvalues at different time intervals [i.e., $\left.430 \gamma_{1}^{2}(\tau)\right]$ then form a trajectory that indicates the modal interferences and variance changes of the 431 system in the absence of stochastic forcing. Since the dynamical operator of the LIM is nonnormal 432 (i.e., $\mathbf{L}^{\mathbf{T}} \mathbf{L} \neq \mathbf{L L}^{\mathbf{T}}$ ), it allows the resultant damping modes to interact with each other and contribute 433 to the transient amplification of the variance without the presence of stochastic forcing. This 434 provides a perspective to, for example, interpret errors in the initial conditions and understand the 435 error growth of the system. In the present LIM, the largest transient amplification of the SST 436 variance was found at 9 months.

437 The optimal precursor indicates the initial condition that maximises the growth of the state vector 438 over a specified time interval $\tau$ ( $\tau=9$ months in this case). The optimal precursor is derived as the 439 corresponding leading eigenvector of $\mathbf{G}^{\mathrm{T}}(\tau) \mathbf{G}(\tau)$ when $\tau=9$ months is specified. 


\section{Data availability}

441 The atmospheric data used in this work are freely available from

442 https://psl.noaa.gov/data/gridded/data.ncep.reanalysis.pressure.html. The model configurations

443 and model outputs used in this work are available on request from TJO. The AR1 and LIM codes

444 are available on request from JL.

445

446

447 Acknowledgements

$448 \mathrm{JL}$ is grateful for a PhD scholarship provided by the ARC Centre of Excellence for Climate System 449 Science (CE110001028), a University of Tasmania tuition fee scholarship, and a CSIRO-UTAS

450 Quantitative Marine Science top-up scholarship [including support from the Australian

451 Commonwealth Scientific Research Organisation (CSIRO) Postgraduate scheme]. TJO was

452 supported by the CSIRO Decadal Climate Forecasting Project (https://research.csiro.au/dfp). NJH

453 acknowledges funding support from the ARC Centre of Excellence for Climate Extremes

454 (CE170100023) and the Australian Government National Environmental Science Programme 455 Climate Systems Hub.

\section{Author contributions}

458 JL conceived the work, carried out the data analyses and wrote most of the paper. TJO supplied 459 the ACCESS-O model outputs. TJO and NJH contributed to the interpretation of the model results 460 and analysis, and also to the text. 


\section{Competing interests}

463 The authors declare no competing interests.

464 